\title{
Absolute scale power measurements of frequency-locked coherent transition radiation
}

\author{
Roark A. Marsh, Amit S. Kesar, and Richard J. Temkin \\ Plasma Science and Fusion Center, Massachusetts Institute of Technology, Cambridge, Massachusetts 02139, USA
}

(Received 15 May 2007; published 7 August 2007)

\begin{abstract}
We report the absolute power measurement of frequency-locked terahertz coherent transition radiation (CTR) from a train of electron bunches produced by a $17.14 \mathrm{GHz}$ accelerator. Frequency measurements were obtained by two independent techniques: a wave meter with a video diode detector, and a double heterodyne system. Good agreement was seen between these two techniques. The emitted radiation was observed to be a comb of frequencies that are harmonics of the $17.14 \mathrm{GHz}$ accelerator frequency. The heterodyne receiver system showed that each of the emitted harmonics has a very narrow bandwidth, about $25 \mathrm{MHz}$, which corresponds to the transform-limited bandwidth for the bunch train length of $40 \mathrm{~ns}$. The highest observed frequency was $377.08 \mathrm{GHz}$, the 22 nd harmonic of the $17.14 \mathrm{GHz}$ accelerator frequency. The emitted CTR power was measured on an absolute scale to test the theory of CTR. The power was compared with calculations made using an electric field integral equation (EFIE) formulation and excellent agreement was obtained. This agreement serves as a valuable benchmark of the EFIE code, proof of both its validity and usefulness.
\end{abstract}

DOI: 10.1103/PhysRevSTAB.10.082801

PACS numbers: 41.60.- m, 52.70.Gw, 29.27.Fh

\section{INTRODUCTION}

Transition radiation is the name given to electromagnetic radiation produced when a charge transits across an interface between media with different electrical properties. For a transition from vacuum into an infinite conducting half plane, radiation is emitted back into the vacuum region. For a transition from vacuum through a foil, radiation is produced both from the transit into and out of the foil, as illustrated in Fig. 1. Transition radiation has a thorough theoretical foundation [1-4], and has found many applications both as a $\mathrm{THz}$ radiation source $[5,6]$ and as a beam diagnostic $[7,8]$.

Transition radiation from bunched electron beams has an additional benefit in terms of radiation production: for wavelengths longer than the length of the electron bunch, the radiation produced by the individual charges is coherent (in phase), so that the fields add, and thus the power scales with the number of charges squared. This coherent transition radiation (CTR) allows bunched beams to produce intense radiation. For a beam produced from an $\mathrm{rf}$ linac utilizing a DC injector, bunches are spaced by a length of time corresponding to the inverse of the rf frequency. A train of bunches with regular spacing will produce radiation corresponding to the Fourier transform of the train. A single bunch produces a characteristic broad power spectrum vs frequency based on the transform of the individual bunch. The power spectrum of a train of bunches has the same overall frequency bandwidth, but is constructed from peaks at integer multiples of the inverse of the bunch spacing in time (which corresponds to the frequency of the accelerator that produced the bunches). The width in frequency of each peak is inversely proportional to the bunch train length. The resulting power enhancement at integer multiples of the $\mathrm{rf}$ frequency is an example of frequency locking.

All of these enhancements to the total power can make CTR an attractive radiation source, as it can be scaled to high power. CTR can be used to generate short radiation pulses for use as small time scale probes by using short electron bunches. CTR can also be used as a beam diagnostic. Autocorrelation can be used to determine bunch length by extracting the radiation into the arms of an interferometer; the bunch length corresponds to the width of the interference as the optical path length difference in the two arms is varied [7]. Electro-optic techniques can also be used for sampling short pulses [8].

The CTR theory used in this paper is based on the electric field integral equation (EFIE) method developed, and reported in [9-11]. Maxwell's equations are solved in the frequency domain in an integral formulation, taking

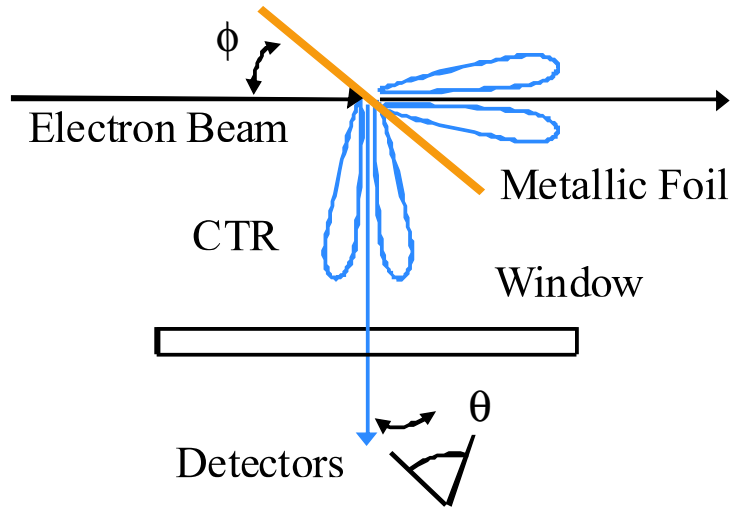

FIG. 1. (Color) Schematic of the experimental setup; $\phi=45^{\circ}$ is the orientation angle of the foil with respect to the beam, and $\theta$ is the observation angle. 
into account the boundary conditions imposed by the presence of conducting media with an arbitrary twodimensional profile. A Green's function is used to calculate the fields from a single charge, which are then extended to a three-dimensional bunch profile. Power calculations follow straightforwardly from field calculations. The EFIE code used was originally developed to more accurately predict and interpret Smith-Purcell radiation (SPR); radiation produced when a beam passes above a periodic metallic structure, such as a grating. Very good agreement has been achieved by comparing SPR power measurements on an absolute scale with the EFIE code. Predicted absolute power levels have been observed with order of magnitude agreement and 30\% errors [12]. Absolute power level measurements on CTR will be used to further validate the EFIE method.

Frequency locking has been seen in observations of SPR [13], and similar techniques were used to observe it in CTR. Previously, bunch to bunch interference has been observed in synchrotron, transition, and diffraction radiation by using optical path length differences, or power spectra. Optical path length difference techniques observe interference, but only for single bunch interference. For a given path length difference an interference pattern is observed; when the path length distance is increased by exactly the length between adjacent bunches, the pattern repeats $[14,15]$. Such interference has been observed in transition radiation [14], as well as in synchrotron radiation [15]. High resolution power spectra see fine structure in the frequency content of the radiation. Rather than appearing flat or smooth, peaks can be resolved at integer multiples of the rf frequency [15]. Such spectra have been observed using synchrotron radiation, though the resolution is on the order of the rf frequency, or the inverse of the bunch spacing. By operating at higher frequency and using higher resolution techniques we can get a better picture of this effect.

In this paper we report measurements of frequencylocked CTR with several innovations: calibrated diode detectors are used to compare power with an EFIE theory on an absolute scale, and frequency locking is observed with very high precision.

\section{EXPERIMENTAL SETUP}

The bunch train used in these radiation experiments was created with the $17 \mathrm{GHz}$ linac manufactured by Haimson Research Corporation and installed at MIT. The parameters of the linac are summarized in Table I.

The linac is powered by a single high power modulator [16], which produces $500 \mathrm{kV}$ microsecond flattop pulses for both the linac DC thermionic gun, and a $17.14 \mathrm{GHz}$ klystron [17]. Beam current and size are controlled by focusing the beam with solenoidal lenses prior to collimation. The DC beam is bunched prior to linac injection using an rf chopper and prebuncher system [18]. The system was
TABLE I. Operating parameters.

\begin{tabular}{lc}
\hline \hline rf frequency & $17.14 \mathrm{GHz}$ \\
Beam energy & $18 \mathrm{MeV}$ \\
Bunch length & $1 \mathrm{ps}$ \\
Bunch charge & $4.6 \mathrm{pC}$ \\
Foil dimensions & $5 \mathrm{~cm} \times 5 \mathrm{~cm}$ \\
\hline \hline
\end{tabular}

operated in a long pulse mode, in which the DC beam is primarily prebunched, with low chopper power operating only to remove a low energy tail from the bunch; this produced $1 \mathrm{ps}$ bunches, as verified using a circularly polarized beam deflector bunch length diagnostic [19,20]. The linac is a constant gradient traveling-wave structure consisting of 94 cells operating in a $2 \pi / 3$ mode, providing a beam energy of $18 \mathrm{MeV}$. An rf pulse length of $100 \mathrm{~ns}$ corresponds to a bunch train of $\sim 40 \mathrm{~ns}$ because of the $60 \mathrm{~ns}$ fill time of our accelerator structure. In previous work, a large uncertainty in beam current led to large experimental errors [21]. The chopper and prebuncher injection system was optimized to provide a stable pulse current of $78 \mathrm{~mA}$, corresponding to a bunch charge of $4.6 \mathrm{pC}$. A toroidal lens follows the linac where the beam is focused to an emittance limited spot size of $1 \mathrm{~mm}$. This size was verified by beam interception measurements. The beam was then passed through a transition radiation experimental chamber, and into a Faraday cup.

CTR was produced by passing the beam through a $5 \mathrm{~cm}$ square, $25 \mu \mathrm{m}$ thick titanium foil placed at $45^{\circ}$ with respect to the beam, as shown in Fig. 1. Radiation was then produced at $90^{\circ}$ with respect to the beam, and extracted though a fused quartz window. The angle $\theta$ is defined as the deviation of the observation point from $90^{\circ}$ to the beam, in the plane defined by the beam and foil normal. To maintain its position, the foil was mounted on a frame which was attached to a vacuum push/pull feedthrough so that the foil could be centered with respect to the beam.

The majority of power measurements were made using a WR6 (110 to $170 \mathrm{GHz}$ band) broadband detector manufactured by Pacific Millimeter Products, which consisted of a single GaAs zero bias diode. The video diode detector was mounted with remote pitch/yaw control on an adjustable tripod with a WR6 horn antenna. The horn and diode were calibrated using a $140 \mathrm{GHz}$ source. The diode was then scanned in both elevation and across the window to determine the angular distribution of the radiation. The distance between the foil and the horn antenna was kept constant, at $0.94 \mathrm{~m}$, for all angles of observation.

Two methods were used to measure frequency locking in CTR. The first consisted of a frequency calibrated Hughes $45718 \mathrm{H}-1000$ wave meter (a tunable band-stop filter with a high quality factor) connected between the horn antenna and the WR6 diode. The second method used a double heterodyne receiver, as in [13], shown schematically in Fig. 2. 


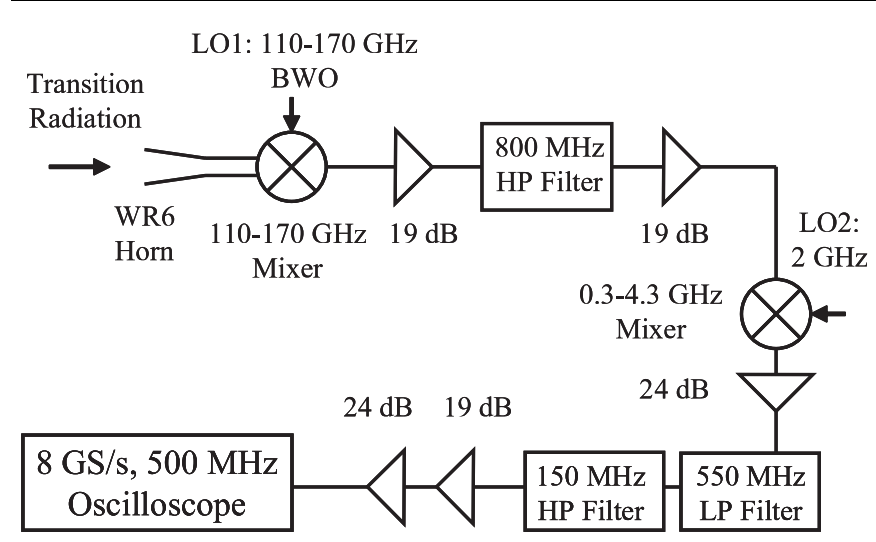

FIG. 2. Double heterodyne system schematic. Incoming radiation is mixed twice with local oscillators, filtered, amplified, and then read as FFT peaks on an oscilloscope.

The basic principle of a heterodyne receiver is to mix two different frequency signals together and observe the lower frequency beats of the mixed signal. In this case, an unknown frequency signal, the CTR radiation, was mixed with a multiple, $n$, of a known local oscillator at frequency $f_{\mathrm{LO} 1}$ to produce a first intermediate frequency (IF) signal. This IF signal was then mixed with a second local oscillator of frequency $f_{\mathrm{LO} 2}$ to produce a second IF signal. Analysis of these signals allowed higher harmonics to be observed, and distinguished. A double heterodyne system was used because of the relative closeness of the many frequency-locked rf signals (spaced every $17.14 \mathrm{GHz}$ ); a second mixing was used in order to observe a single peak on the fast-Fourier-transform (FFT), rather than several overlapping ones. Because of the nature of the harmonic mixing, for each observed CTR frequency, four FFT peaks were observed, corresponding to

$$
f_{\mathrm{RF}}=n f_{\mathrm{LO} 1} \pm f_{\mathrm{LO} 2} \pm \Delta,
$$

where $\Delta$ is the observed frequency offset. The order $n$ was determined by changing the LO1 frequency and observing how quickly the FFT peak frequency $\left(f_{\mathrm{RF}}\right)$ changed. The LO1 frequency was based on the frequency calibration of the backward wave oscillator (BWO) source, and the $\mathrm{LO} 2$ frequency was fixed at $2 \mathrm{GHz}$. The actual rf frequency was measured by averaging over the four signals that corresponded to each frequency signal. The system has a very narrow bandwidth of less than $10 \mathrm{MHz}$, allowing measurements to be made to the bandwidth limit inherent in the incoming CTR signal.

Loss measurements were essential in understanding, and measuring the absolute CTR power. The $12.7 \mathrm{~mm}$ thick fused quartz vacuum window transmission was cold tested using an E8363B Agilent Precision Network Analyzer with Olsen Microwave Lab millimeter wave extenders. The real part of the refractive index ranged from 1.85 to 1.90 , and the loss tangent was $10^{-4}$, allowing an overall transmission coefficient to be calculated.

\section{EXPERIMENTAL RESULTS}

Coherence was verified by observing the CTR power detected with the WR6 diode as the beam current was varied using the injector lenses, while keeping the other linac parameters fixed. Figure 3 shows the measured power vs the square of the pulse current. The fit shown by a solid line indicates that the power scales as the square of the current, showing that the emitted radiation is coherent. The maximum point, $\sim 200 \mathrm{~mA}$, corresponds to the point at which significant beam loading begins to affect bunch acceleration.

The absolute angular distribution of CTR power (in milliwatts), as measured with the WR6 band horn and detector, is shown in Fig. 4 as a function of the emitted angle relative to 90 degrees, where the angle $\theta$ is defined in Fig. 1. The measured CTR power is compared with the predicted power from the EFIE code, which is shown by a solid line. The EFIE code was run for all harmonics in the WR6 receiver band (mainly $110-170 \mathrm{GHz}$ ), using the beam parameters in Table I. It was used to calculate the emitted power at the location of the receiver, $r=0.94 \mathrm{~m}$ from the foil. The predicted power was corrected for the frequency dependence of the absorption in the fused quartz window and the effective aperture, $A_{e}$, of the WR6 band receiver (horn antenna and detector). The effective aperture of the horn is given by the formula

$$
A_{e}=G_{h} \frac{\lambda^{2}}{4 \pi}
$$

where $G_{h}$ is the horn gain which varies slightly with frequency, from 22.3 to $23.4 \mathrm{~dB}$. Error bars on the observed absolute power are the result of combined errors from current measurements, statistical variation of the diode signal, and an estimation of the systematic error based on background signals. Excellent agreement is obtained between the EFIE code results and the experiment. In previous work, we had reported good qualitative agreement between theory and experiment, but poor (factor of $\sim 3$ )

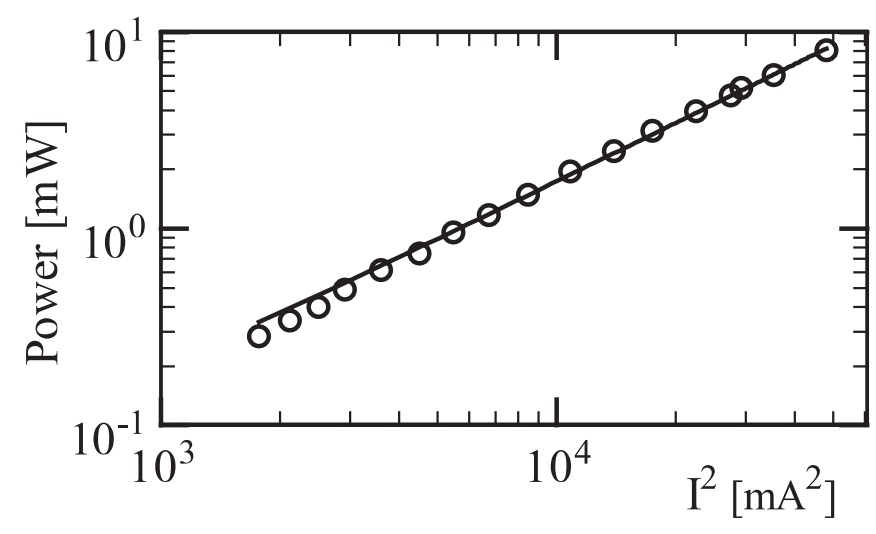

FIG. 3. CTR power measured by the diode as a function of the pulse current squared. The solid line is a linear fit to the data points. 


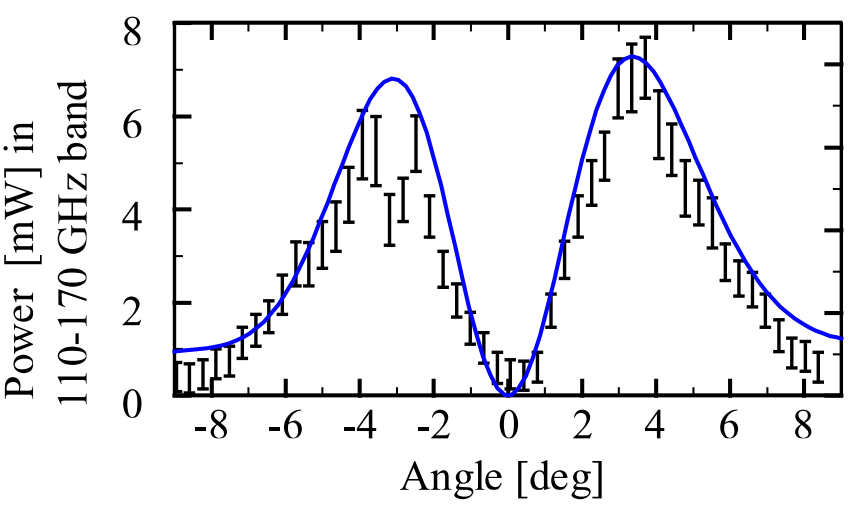

FIG. 4. (Color) Angular distribution of CTR power measured by the diode with error bars, and theory calculations made with EFIE code, shown by the solid line. The angular error on each data point is \pm 0.2 degrees.

quantitative agreement [21]. The present experiments have been greatly improved by taking more extensive data, using more precise current measurements, and operating under optimal linac conditions.

Wave meter measurements, shown in Fig. 5, show a dip (or reduction in microwave power transmitted through the wave meter) near $120 \mathrm{GHz}$, which is near the 7th harmonic $(119.98 \mathrm{GHz})$ of the $17.14 \mathrm{GHz}$ accelerator frequency. The wave meter dip indicates that the microwave power spectrum has frequency content at the frequency (near $120 \mathrm{GHz}$ ) shown on the wave meter. Similar dips were seen near 137 and $154 \mathrm{GHz}$, corresponding to the 8th and 9 th harmonics of the accelerator frequency, respectively. The bandwidth of the observed dips was $\sim 100 \mathrm{MHz}$ which corresponds to the resolution of the wave meter. The actual bandwidth was measured with heterodyne receiver measurements, discussed below. Wave meter dips were used as a convenient and rapid means of determining the frequency content of the CTR over a wide range of frequencies. These measurements confirmed that the emitted radiation was

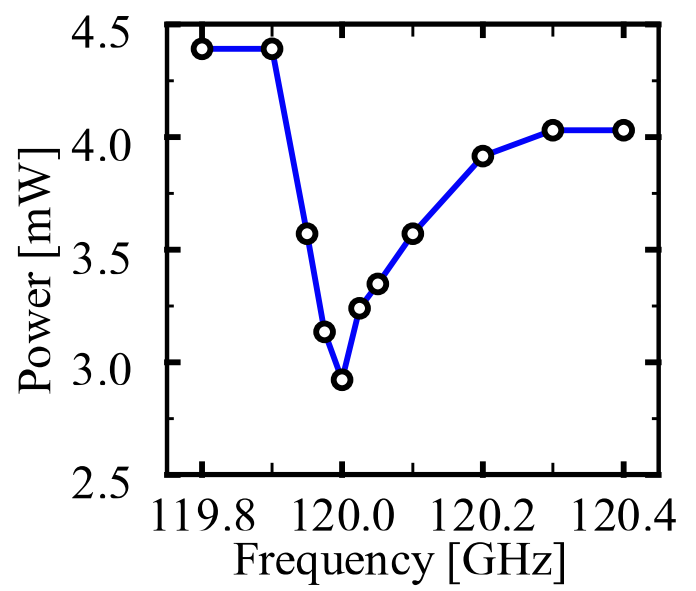

FIG. 5. (Color) Power measured by the diode as a function of the wave meter dial reading. The dip shown at $120.0 \pm 0.025 \mathrm{GHz}$ is consistent with the 7 th harmonic of the rf frequency.

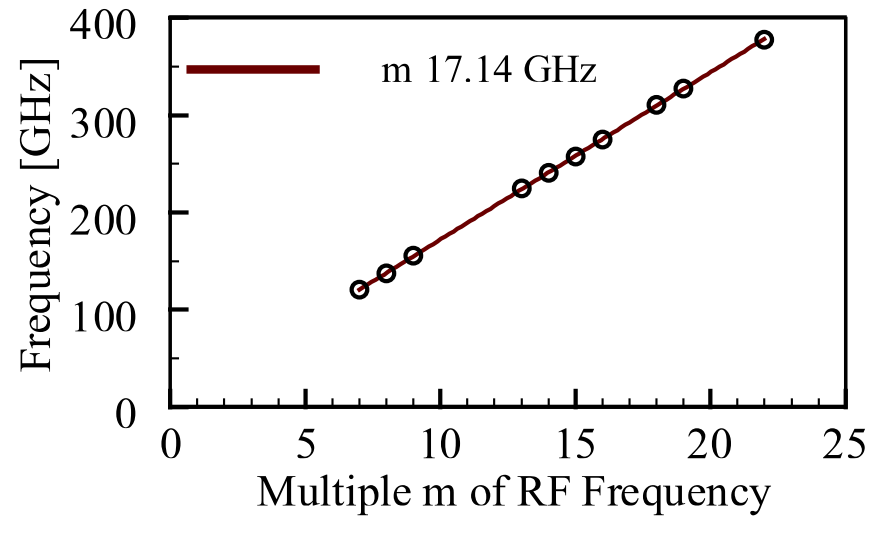

FIG. 6. (Color) CTR frequencies measured with the double heterodyne receiver, open circles. Radiation is only observed at integer harmonics of the rf frequency. The straight line is a fit to the data and has a slope consistent with the accelerator frequency of $17.14 \mathrm{GHz}$.

frequency-locked: only observed at harmonics of the accelerator drive frequency of $17.14 \mathrm{GHz}$.

Frequency locking was further investigated using double heterodyne receiver measurements. For each observed CTR frequency, four FFT peaks were observed, and averaged to find the frequency of the radiation. A summary of results is given in Fig. 6. Signals are found only at integer harmonics of the rf frequency. The specific harmonics observed were in the range from the 7 th through the 22nd. Some harmonics could not be observed because the available microwave equipment did not generate the required LO signal. The highest harmonic observed, the 22nd, at $377.08 \mathrm{GHz}$ corresponds to a radiation signal limited by the receiver sensitivity. Transition radiation has been used as a bunch length diagnostic due to the relatively flat frequency spectrum, which allows the coherent structure of CTR to be observed. The drop off of higher

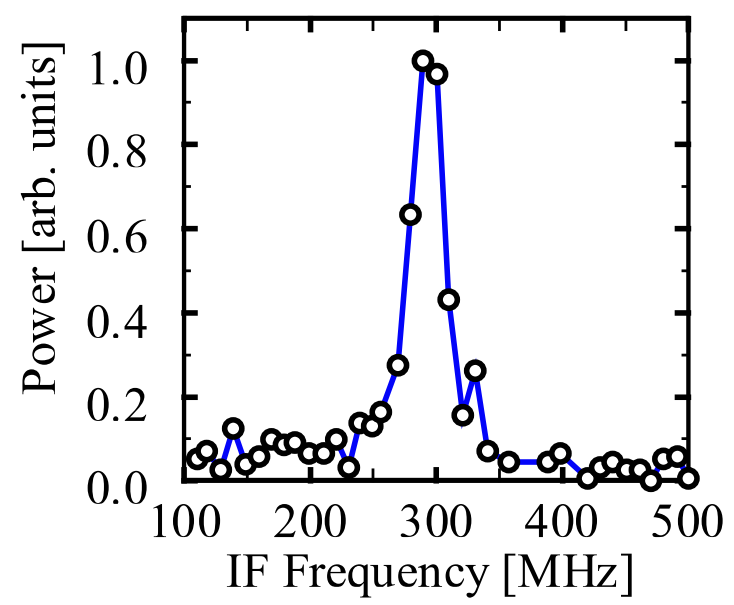

FIG. 7. (Color) FFT of the IF frequency from the double heterodyne receiver operating near $240 \mathrm{GHz}$, the 14th harmonic of the accelerator frequency. A narrow bandwidth of $28 \mathrm{MHz}$ FWHM is observed. 
harmonics below detection threshold can be used to estimate the bunch length. An estimated bunch length of $1 \pm$ $0.2 \mathrm{ps}$ is in agreement with measurements made using independent methods. A typical FFT peak is shown in Fig. 7, exhibiting a very narrow bandwidth of $28 \mathrm{MHz}$ at $240 \mathrm{GHz}$ (the 14th harmonic of $17.14 \mathrm{GHz}$ ); this width corresponds to $\sim 600$ bunches (or about $35 \mathrm{~ns}$ ) in the train.

\section{DISCUSSION AND CONCLUSIONS}

The experimentally observed CTR angular distribution, coherence, and frequency locking were in agreement with theoretical expectations. The angular shape fits theory well, both in terms of qualitative shape and absolute power level. The EFIE code was able to accurately predict how much power was observed. These power measurements are novel and important because they were compared with theory on an absolute scale. Frequency locking was verified by two independent measurements: one made with a wave meter, and another using a double heterodyne receiver system. The wave meter measurements verified high power content in the rf harmonics within its frequency range. The heterodyne system was able to resolve frequency content with very narrow bandwidth, up to the 22nd harmonic of the rf frequency, a frequency of $377.08 \mathrm{GHz}$. Previous observations of bunch to bunch coherence, or frequency locking, have been of two types: interferometer measurements, in which single bunch interference is observed; and high resolution spectra, limited by their frequency resolution. With the techniques described here, we have been able to very clearly observe the frequency locking of multiple bunches with very high resolution, due to the sensitivity of the heterodyne system and the high operating rf frequency $(17.14 \mathrm{GHz})$ of the linac. The EFIE code was also further benchmarked as an accurate means of predicting radiation from metallic structures. Accurate validation of the absolute power levels predicted by the code and observed in the lab is vital for absolute power based bunch length diagnostics, as proposed in [11].

\section{ACKNOWLEDGMENTS}

This work was supported by the Department of Energy, Division of High Energy Physics Contract No. DE-FG0291ER40648. The authors gratefully acknowledge the help of Ivan Mastovsky, Bill Mulligan, and Jagadishwar Sirigiri.

[1] V. L. Ginzburg and I. M. Frank, Sov. Phys. JETP 16, 15 (1946).

[2] F. G. Bass and V. M. Yakovenko, Sov. Phys. Usp. 8, 420 (1965).
[3] V.L. Ginzburg and V. N. Tsytovich, Transition Radiation and Transition Scattering (Adam Hilger, New York, 1990).

[4] Y. Takakura and O. Haeberlé, Phys. Rev. E 61, 4441 (2000).

[5] A. N. Chu, M. A. Piestrup, T.W. Barbee, Jr., and R.H. Pantell, J. Appl. Phys. 51, 1290 (1980).

[6] W. P. Leemans, C. G. R. Geddes, J. Faure, Cs. Tóth, J. van Tilborg, C. B. Schroeder, E. Esarey, G. Fubiani, D. Auerbach, B. Marcelis, M. A. Carnaham, R. A. Kaindl, J. Byrd, and M.C. Martin, Phys. Rev. Lett. 91, 074802 (2003).

[7] P. Muggli, M. J. Hogan, C. D. Barnes, D. Walz, P. Krejcik, R. H. Siemann, H. Schlarb, and R. Ischebek, in Proceedings of the 2005 Particle Accelerator Conference (IEEE, Piscataway, NJ, 2005), pp. 4102-4104.

[8] J. van Tilborg, C.B. Schroeder, C. V. Filip, Cs Tóth, C. G. R. Geddes, G. Fubiani, R. Huber, R. A. Kaindl, E. Esarey, and W. P. Leemans, Phys. Rev. Lett. 96, 014801 (2006).

[9] O. Haeberlé, P. Rullhusen, J. M. Salomé, and N. Maene, Phys. Rev. E 49, 3340 (1994).

[10] A. S. Kesar, M. Hess, S. E. Korbly, and R. J. Temkin, Phys. Rev. E 71, 016501 (2005).

[11] A.S. Kesar, Phys. Rev. ST Accel. Beams 8, 072801 (2005).

[12] A. S. Kesar, R. A. Marsh, and R. J. Temkin, Phys. Rev. ST Accel. Beams 9, 022801 (2006).

[13] S. E. Korbly, A. S. Kesar, J. R. Sirigiri, and R. J. Temkin, Phys. Rev. Lett. 94, 054803 (2005).

[14] T. Takahashi, Y. Shibata, F. Arai, K. Ishi, T. Ohsaka, M. Ikezawa, Y. Kondo, T. Nakazato, S. Urasawa, R. Kato, S. Niwano, and M. Oyamada, Phys. Rev. E 48, 4674 (1993).

[15] Y. Shibata, T. Takahashi, K. Ishi, F. Arai, H. Mishiro, T. Ohsaka, M. Ikezawa, Y. Kondo, S. Urasawa, T. Nakazato, R. Kato, S. Niwano, and M. Oyamada, Phys. Rev. A 44, R3445 (1991).

[16] W. J. Mulligan, S. C. Chen, G. Bekefi, B. G. Danly, and R.J. Temkin, IEEE Trans. Electron Devices 38, 817 (1991).

[17] J. Haimson, B. Mecklenburg, G. Stowell, K. E. Kreischer, and I. Mastovsky, in Proceedings of the 1999 Conference on High Energy Density Microwaves, AIP Conf. Proc. No. 474 (AIP, New York, 1999), p. 137.

[18] J. Haimson and B. Mecklenburg, in Proceedings of the 1995 Particle Accelerator Conference (IEEE, Piscataway, New Jersey, 1995) pp. 755-757.

[19] J. Haimson, B. Mecklenburg, G. Stowell, and B. Ishii, in Advanced Accelerator Concepts: Tenth Workshop, AIP Conf. Proc. No. 647 (AIP, New York, 2002), pp. 810-820.

[20] J. Haimson, in Proceedings of the 2004 Advanced Accelerator Concepts Conference, AIP Conf. Proc. 737 (AIP, New York, 2004), pp. 95-108.

[21] R. A. Marsh, A. S. Kesar, and R. J. Temkin, in Proceedings of the 2005 Particle Accelerator Conference (IEEE, Piscataway, NJ, 2005), pp. 452-454. 